\title{
A green solventless protocol for Michael addition of phthalimide and saccharin to acrylic acid esters in the presence of zinc oxide as a heterogeneous and reusable catalyst
}

\author{
Abdolkarim Zare, ${ }^{* a}$ Alireza Hasaninejad, ${ }^{* b}$ Ali Khalafi-Nezhad, ${ }^{c}$ \\ Ahmad R. Moosavi Zare, ${ }^{c}$ Abolfath Parhami, ${ }^{c}$ and Gholam R. Nejabat ${ }^{c}$ \\ ${ }^{a}$ Department of Chemistry, Payam Noor University of Bushehr, Bushehr 1698, Iran \\ ${ }^{b}$ Department of Chemistry, Faculty of Science, Persian Gulf University, Bushehr 75169, Iran \\ ${ }^{c}$ Department of Chemistry, College of Science, Shiraz University, Shiraz 71454, Iran \\ E-mail: abdolkarimzare@yahoo.com, ahassaninejad@yahoo.com
}

\begin{abstract}
A very simple and efficient solvent-free procedure for the synthesis of $\mathrm{N}$-alkyl derivatives of phthalimide and saccharin is described. Michael addition of phthalimide and saccharin to acrylic acid esters in the presence of catalytic amount of zinc oxide $(\mathrm{ZnO})$ and tetrabutylammonium bromide (TBAB) under microwave and thermal conditions affords the title compounds. The advantages of this method are high yields, short reaction times, low cost and matching with green chemistry protocols.
\end{abstract}

Keywords: Michael addition, phthalimide, saccharin, acrylic acid ester, solventless, zinc oxide

\section{Introduction}

Michael addition of phthalimide and saccharin to $\alpha, \beta$-unsaturated esters is significant as this reaction provides a simple and appealing route toward synthesis of $\mathrm{N}$-alkyl derivatives of these compounds. Some $N$-alkyl phthalimides have been applied as antipsychotic, ${ }^{1}$ anti-inflammatory agents, ${ }^{2}$ and receptors. ${ }^{3}$ Hypolipidemic activity was also reported for phthalimide derivatives. ${ }^{4}$ Saccharin and its derivatives are important in medicinal chemistry. ${ }^{5}$ Furthermore, saccharin can complex with various metal ions, such as $\mathrm{Mg}^{2+}, \mathrm{Ca}^{2+}, \mathrm{Sr}^{2+}, \mathrm{Pd}^{2+}, \mathrm{Cu}^{2+}$ and etc. ${ }^{6}$ Conjugate addition of phthalimide and saccharin to $\alpha, \beta$-unsaturated esters also affords protected $\beta$-amino acids. This class of compounds are essential components in many bioactive compounds and drugs scaffolds, including $\beta$-peptides, ${ }^{7}$ imeriamine (hypoglycemic and antiketogenic agent, Figure 1), ${ }^{8}$ vitamin $\mathrm{B}_{3}$ (Figure 1), ${ }^{9}$ cryptophycin (antitumor), ${ }^{10}$ and TAN-1057 A (antibiotic). ${ }^{11}$ In aza-conjugate reactions, the nucleophilic nitrogen is usually among the powerful ones, such as amines $^{12}$ which their use in the reaction can lead to side products, such as amides via 
nucleophilic attack of amine to carbonyl group of $\alpha, \beta$-unsaturated esters. ${ }^{13}$ Furthermore, 1,2 and 1,4 condensation of $\beta$-amino residue to $\alpha, \beta$-unsaturated esters cause polymerization or tar formation. ${ }^{13}$ Moreover, many of these procedures often require a large excess of reagents. ${ }^{13}$ Therefore, in this context, using imides instead of amines in Michael addition to $\alpha, \beta$-unsaturated esters seems to be more favorable since lower nucleophilicity of nitrogen leads to less side reactions.

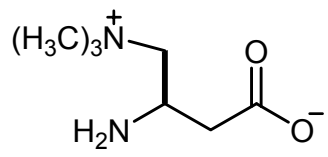

Imeriamine

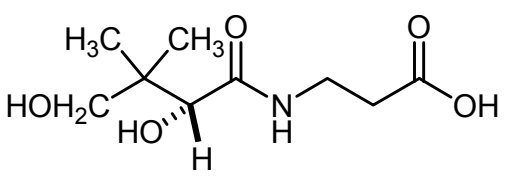

Vitamin $\mathbf{B}_{3}$

Figure 1. The structures of imeriamine and vitamin $B_{3}$.

In the previous methods for aza-Michael addition of imides or their salts to $\alpha, \beta$-unsaturated compounds, reagents such as $\mathrm{Na}$ in absolute ethanol, ${ }^{14 \mathrm{a}} \mathrm{K}_{2} \mathrm{CO}_{3}{ }^{14 \mathrm{~b}}$ and $\mathrm{AlMe}_{2} \mathrm{Cl}^{14 \mathrm{c}}$ have been used. However, these reactions have been performed in solution conditions and in relatively long reaction times. To the best of our knowledge, there is no report of Michael addition of imides to $\alpha, \beta$-unsaturated esters under solvent-free conditions.

Recently, mineral oxides have proved to be useful to chemists in the laboratory and industry due to the good activation of adsorbed compounds and reaction rate enhancement, selectivity, easier workup and recyclability of the supports and the eco-friendly reaction conditions. ${ }^{15,16}$ Zinc oxide $(\mathrm{ZnO})$ is certainly one of the most interesting of these oxides because it has surface properties that suggest that a very rich organic reactions may occur there. ${ }^{16}$ Zinc oxide is an inexpensive, moisture stable, reusable, commercially available and environmentally benign catalyst. This catalyst has been used in several transformations, such as Beckmann rearrangements, ${ }^{16 \mathrm{a}}$ Friedel-Crafts acylation, ${ }^{16 \mathrm{~b}}$ benzylic oxidations, ${ }^{16 \mathrm{c}}$ conversion of oximes to nitriles, ${ }^{16 \mathrm{~d}}$ synthesis of cyclic ureas, ${ }^{16 \mathrm{e}}$ and acylation of alcohols and amines. ${ }^{16 \mathrm{f,g}}$

Although a few methods to achieve Michael reaction of imides are known, newer methods continue to attract attention for their experimental simplicity and effectiveness. The coupling of microwave irradiation with the use of catalysts or mineral-supported reagents provides chemical processes with special attributes, such as enhanced reaction rates, higher yields, better selectivity and improved ease of manipulation. ${ }^{17}$

Along with our previous works on aza-Michael reactions ${ }^{18}$ and also in extension of our previous studies on application of solvent-free technology in organic synthesis, ${ }^{18,19}$ herein we report a clean, facile and rapid solventless method for Michael addition of phthalimide and saccharin to acrylic acid esters in the presence of catalytic amount of zinc oxide and tetrabutylammonium bromide (TBAB) under microwave and thermal conditions (Schemes 1 and 2). 


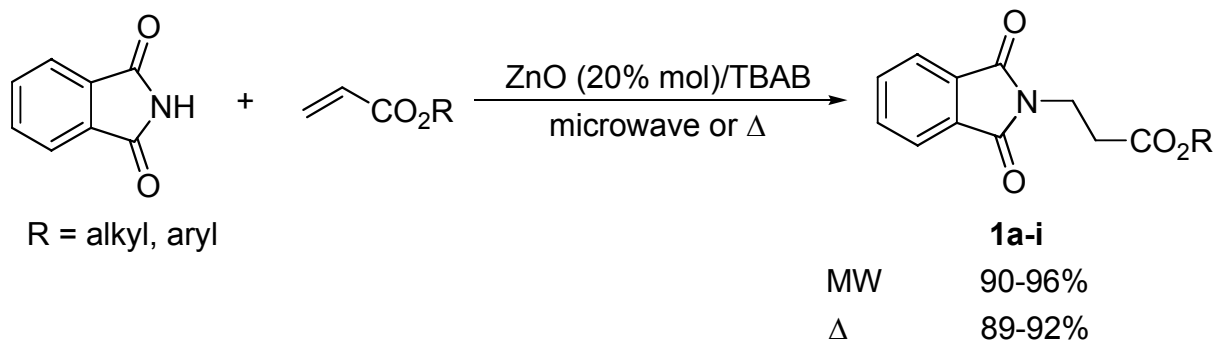

Scheme 1. Michael addition of phthalimide to acrylic acid esters.

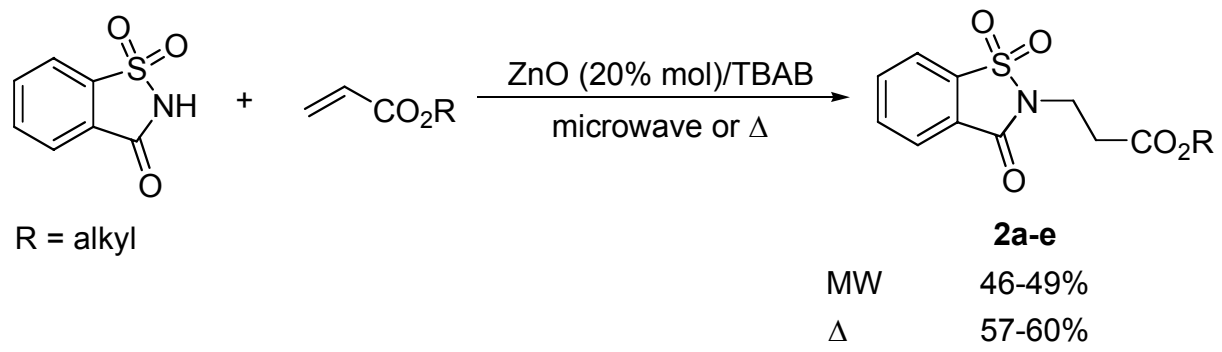

Scheme 2. Michael addition of saccharin to acrylic acid esters.

\section{Results and Discussion}

To obtain the optimized reaction conditions, we have studied the reaction of phthalimide with $n$ butyl acrylate as a model reaction to provide compound $1 \mathrm{~b}$ (Table 3). For this purpose, at first, the effect of various basic catalysts was examined under microwave and thermal conditions to evaluate their capabilities. The results are summarized in Table 1. As it is shown in Table 1 (entry 1), higher yield in shorter reaction time were obtained in both conditions when zinc oxide was used. Therefore, zinc oxide was chosen as catalyst for all reactions.

In order to select the appropriate microwave power, the model reaction was examined at different microwave powers $(100-600 \mathrm{~W})$ with controlled temperature $\left(\max .130{ }^{\circ} \mathrm{C}\right)$ in the presence of $\mathrm{ZnO}$ and $\mathrm{TBAB}$. The best results were obtained at $300 \mathrm{~W}$. The reaction was also examined at $60-130{ }^{\circ} \mathrm{C}$ in thermal conditions. Higher yield and shorter reaction time were attained at $100^{\circ} \mathrm{C}$.

In another study, the role of TBAB was evaluated in both conditions. The absence of TBAB in the reaction media gave low reaction yield even by prolonging the reaction time or enhancing the microwave power and the temperature. Thus, the presence of TBAB in our reaction is critically significant. TBAB melts at $100{ }^{\circ} \mathrm{C}$ and creates a homogeneous reaction media whose resemblance is not far from that of ionic liquid. ${ }^{18 \mathrm{a}, 19 \mathrm{c}, 20}$ In addition, in microwave conditions, 
TBAB absorbs the microwave irradiation as well as generates in situ heat and increases the temperature higher than its melting point $\left(100-103{ }^{\circ} \mathrm{C}\right) .{ }^{18 \mathrm{a}, 19 \mathrm{c}, 20}$

To compare the efficiency of the solvent-free versus solution conditions, the reaction was examined in several solvents under microwave and thermal conditions. Thus, a mixture of phthalimide $(2 \mathrm{mmol}), \mathrm{ZnO}(0.4 \mathrm{mmol})$ and $n$-butyl acrylate $(3 \mathrm{mmol})$ was irradiated in microwave oven $\left(300 \mathrm{~W}\right.$, max. $\left.130{ }^{\circ} \mathrm{C}\right)$ or heated in an oil bath $\left(100{ }^{\circ} \mathrm{C}\right)$ in different solvents $(5$ $\mathrm{mL})$. The results are depicted in Table 2. As it is clear from Table 2, lower yields and longer reaction times were obtained in solution conditions. Therefore, the solvent-free method is more efficient.

Table 1. The effect of different catalysts $(20 \% \mathrm{~mol})$ on reaction of phthalimide $(2 \mathrm{mmol})$ with $n$ butyl acrylate $(3 \mathrm{mmol})$ in the presence of TBAB $(1 \mathrm{mmol})$ under microwave $(\mathrm{MW}, 300 \mathrm{~W}$, max. $\left.130^{\circ} \mathrm{C}\right)$ and thermal $\left(\Delta, 100^{\circ} \mathrm{C}\right)$ conditions

\begin{tabular}{cccccc}
\hline \multirow{2}{*}{ Entry } & \multirow{2}{*}{ Base } & \multicolumn{3}{c}{ Time $(\mathrm{min})$} & \multicolumn{2}{c}{ Yield $^{\mathrm{a}}(\%)$} \\
\cline { 3 - 6 } & & $\mathrm{MW}$ & $\Delta$ & $\mathrm{MW}$ & $\Delta$ \\
\hline 1 & $\mathrm{ZnO}$ & 8 & 60 & 96 & 92 \\
2 & $\mathrm{Cs}_{2} \mathrm{CO}_{3}$ & 10 & 90 & 71 & 66 \\
3 & $\mathrm{~K}_{2} \mathrm{CO}_{3}$ & 12 & 120 & 62 & 50 \\
4 & $\mathrm{MgO}$ & 15 & 150 & 22 & 10 \\
5 & $t$-BuOK & 10 & 90 & 65 & 61 \\
6 & DABCO & 10 & 120 & 43 & 39 \\
7 & 1-Methylimidazole & 10 & 120 & 55 & 52 \\
\hline
\end{tabular}

${ }^{\mathrm{a}}$ Isolated yield.

To investigate the versatility and capacity of our method, the reactions of phthalimide and saccharin were examined with various acrylic acid esters under both microwave and thermal conditions (Table 3). As Table 3 indicates, the reactions proceeded efficiently and the desired Michael adducts were obtained in good to excellent yields. 
Table 2. Comparative synthesis of compound $\mathbf{1 b}$ using solution versus the solvent-free conditions under microwave $\left(\mathrm{MW}, 300 \mathrm{~W}, \max .130^{\circ} \mathrm{C}\right)$ and thermal $\left(\Delta, 100^{\circ} \mathrm{C}\right)$ conditions

\begin{tabular}{cccccc}
\hline \multirow{2}{*}{ Entry } & \multirow{2}{*}{ Solvent } & \multicolumn{3}{c}{ Time $(\min )$} & \multicolumn{2}{c}{ Yield $^{\mathrm{a}}(\%)$} \\
\cline { 2 - 6 } & & MW & $\Delta$ & MW & $\Delta$ \\
\hline 1 & DMSO & 20 & 900 & 67 & 47 \\
2 & DMF & 20 & 900 & 46 & 40 \\
3 & HMPTA & 25 & 900 & 35 & 35 \\
4 & $o$-Xylene & 25 & 900 & 19 & 19 \\
$5^{\text {b }}$ & {$[$ bmim]Br } & 8 & 60 & 93 & 88 \\
$6^{\text {b }}$ & TBAB & 8 & 60 & 96 & 92 \\
\hline
\end{tabular}

${ }^{a}$ Isolated yield. ${ }^{\mathrm{b}}$ The amounts of 1-butyl-3-methylimidazolium bromide and TBAB for the addition of phthalimide $(2 \mathrm{mmol})$ to $n$-butyl acrylate $(3 \mathrm{mmol})$ was $2 \mathrm{~g}$ and $1 \mathrm{mmol}$ respectively.

Table 3. Michael addition of phthalimide and saccharin to acrylic acid esters in the presence of $\mathrm{TBAB}$ and $\mathrm{ZnO}$ under microwave (MW, $300 \mathrm{~W}$, max. $\left.130^{\circ} \mathrm{C}\right)$ and thermal $\left(\Delta, 100{ }^{\circ} \mathrm{C}\right)$ conditions

\begin{tabular}{|c|c|c|c|c|c|c|}
\hline \multirow{2}{*}{ Entry } & \multirow{2}{*}{ Ester } & \multirow{2}{*}{ Product } & \multicolumn{2}{|c|}{ Time $(\min )$} & \multicolumn{2}{|c|}{ Yield $^{\mathrm{a}}(\%)$} \\
\hline & & & MW & $\Delta$ & MW & $\Delta$ \\
\hline $1^{b}$ & & & 8 & 60 & 95 & 90 \\
\hline 2 & & & 8 & 60 & 96 & 92 \\
\hline 3 & & & 8 & 90 & 96 & 91 \\
\hline 4 & & & 8 & 90 & 95 & 91 \\
\hline 5 & & & 9 & 90 & 95 & 90 \\
\hline
\end{tabular}


6<smiles>C=CC(=O)OC/C=C/c1ccccc1</smiles><smiles>O=C(CCN1C(=O)c2ccccc2C1=O)OC/C=C/c1ccccc1</smiles>

10

120

93

90

7<smiles>C=CC(=O)OCC(O)COc1ccccc1OC</smiles><smiles>C=CC(=O)Oc1ccccc1</smiles>

8

9<smiles>C=CC(=O)Oc1ccc2ccccc2c1</smiles>

10<smiles>C=CC(=O)OCCCC</smiles>

11<smiles>C=CC(=O)OCCCCCCC</smiles>

12<smiles>C=CC(=O)OC/C=C/c1ccccc1</smiles>

13<smiles>C=CC(=O)OC(C)C</smiles>

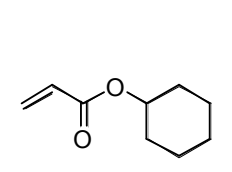<smiles>C=CC(=O)OCC(O)COC(=O)CCN1C(=O)c2ccccc2C1=O</smiles><smiles>O=C(CCN1C(=O)c2ccccc2C1=O)Oc1ccc2ccccc2c1</smiles><smiles>CCCCOC(=O)CCN1C(=O)c2ccccc2S1(=O)=O</smiles><smiles>CCCCCCOC(=O)CCN1C(=O)c2ccccc2S1(=O)=O</smiles><smiles>O=C(CCN1C(=O)c2ccccc2S1(=O)=O)O/C=C/c1ccccc1</smiles><smiles>CC(C)OC(=O)CCN1C(=O)c2ccccc2S1(=O)=O</smiles><smiles>O=C(CCN1C(=O)c2ccccc2S1(=O)=O)OC1CCCCC1</smiles>

10

150

94

89

$4 \quad 30 \quad 92 \quad 90$

$\begin{array}{llll}5 & 45 & 90 & 89\end{array}$

$\begin{array}{llll}2 & 720 & 49 & 60\end{array}$

$\begin{array}{llll}2 & 900 & 48 & 59\end{array}$

$\begin{array}{llll}2 & 900 & 47 & 60\end{array}$

$\begin{array}{llll}2 & 780 & 47 & 59\end{array}$

$2 \quad 900 \quad 46 \quad 57$
14

${ }^{\mathrm{a}}$ Isolated yield. ${ }^{\mathrm{b}}$ In this reaction, the acrylic acid ester/phthalimide (mol $\left./ \mathrm{mol}\right)$ ratio in thermal conditions was $2 / 1$.

To study the structural influence of alkoxy group (-OR) of acrylic acid esters (Michael acceptors) on the reaction, we have investigated the reaction of phthalimide and saccharin with esters containing sterically hindered alkoxy groups under both microwave and thermal 
conditions (Table 3). As it is shown in Table 3, the bulkiness of alkoxy group had no significant effect on yields and reaction times in microwave conditions. In thermal conditions, alkoxy group did not affect the reaction yields; however, longer reaction times were needed when phthalimide or saccharin was introduced to esters possessing sterically hindered alkoxy groups. The reactions of phthalimide with phenolic acrylic acid esters were carried out in shorter reaction times in comparison with other esters in both conditions; but, the yields did not changed (Table 3, entries 8 and 9). Interestingly, in our method, the nucleophilic nitrogen of phthalimide and saccharin does not attack to the carbonyl group of esters. In general, for Michael addition of phthalimide to acrylic acid esters, the microwave method was more efficient in comparison with thermal method. However, for Michael addition of saccharin, thermal conditions gave higher yields.

The interesting behavior of zinc oxide $(\mathrm{ZnO})$ lies in the fact that it can be re-used after simple washing with $\mathrm{CHCl}_{3}$, rendering thus process more economic. The yields of compound $\mathbf{1 b}$ (a model compound) in the $2 \mathrm{nd}, 3 \mathrm{rd}, 4^{\text {th }}$ and $5^{\text {th }}$ uses of the $\mathrm{ZnO}$ were almost as high as in the first use both in microwave and thermal conditions.

\section{Conclusions}

In summary, we have developed an efficient and rapid solvent-free method for Michael addition of phthalimide and saccharin to various acrylic acid esters under microwave and thermal conditions. This new method affords $\mathrm{N}$-alkyl derivatives of phthalimide and saccharin as biologically interesting compounds in short reaction times and excellent yields.

\section{Experimental Section}

General Procedures. All chemicals were obtained from Merck or Fluka chemical companies. The new acrylic acid esters were prepared from acryloyl chloride and alcohols by the reported method ${ }^{16, g}$ and their structures were confirmed by IR, ${ }^{1} \mathrm{H}$ and ${ }^{13} \mathrm{C}$ NMR spectra. The progress of reactions was followed by TLC using silica gel SILG/UV 254 plates. All reactions were carried out using CEM MARS $5^{\mathrm{TM}}$ microwave oven. IR spectra were run on a Shimadzu FTIR-8300 spectrophotometer; $v_{\max }$ in $\mathrm{cm}^{-1}$. The ${ }^{1} \mathrm{H}$ NMR $(250 \mathrm{MHz})$ and ${ }^{13} \mathrm{C}$ NMR $(62.5 \mathrm{MHz})$ were run on a Bruker Avanced DPX-250, FT-NMR spectrometer. Mass spectra were recorded on a Shimadzu GC MS-QP 1000 EX apparatus. Melting points were recorded on a Büchi B-545 apparatus in open capillary tubes and are uncorrected.

Michael addition of phthalimide and saccharin to acrylic acid esters under solvent-free conditions. Microwave Procedure: To a well ground mixture of compounds consisting of imide $(2 \mathrm{mmol}), \mathrm{TBAB}(0.322 \mathrm{~g}, 1 \mathrm{mmol})$ and $\mathrm{ZnO}(0.044 \mathrm{~g}, 0.4 \mathrm{mmol})$ in a microwave vessel was 
added acrylic acid esters $(3 \mathrm{mmol})$ and mixed carefully with a small rod. The mixture was irradiated in the microwave oven at $300 \mathrm{~W}$ for the times reported in Table 3 . The microwave was programmed to give a maximum internal temperature to $130^{\circ} \mathrm{C}$. Afterward, the reaction mixture was cooled to room temperature and suspended in chloroform $(50 \mathrm{~mL})$, filtered and the filtrate was washed with water $(2 \times 40 \mathrm{~mL})$ and dried with $\mathrm{MgSO}_{4}$. The solvent was evaporated and the crude product was purified by column chromatography on silica gel with EtOAc/n-hexane (1/5). Thermal Procedure: To a well ground mixture of imide $(2 \mathrm{mmol})$, TBAB $(0.322 \mathrm{~g}, 1 \mathrm{mmol})$ and $\mathrm{ZnO}(0.044 \mathrm{~g}, 0.4 \mathrm{mmol})$ in a test tube connected to a reflux condenser was added acrylic acid esters ( $3 \mathrm{mmol})$ and mixed carefully with a small rod. The mixture was stirred in an oil bath (100 ${ }^{\circ} \mathrm{C}$ ) for the appropriate time (Table 3). The reaction mixture was cooled to room temperature and suspended in chloroform $(50 \mathrm{~mL})$, filtered and the filtrate was washed with water $(2 \times 40 \mathrm{~mL})$ and dried with $\mathrm{MgSO}_{4}$. The solvent was evaporated and the crude product was purified by column chromatography on silica gel with EtOAc/ $n$-hexane (1/5).

Ethyl 3-phthalimido propanoate (1a). Colorless solid; mp 60-61 ${ }^{\circ} \mathrm{C}$; IR $(\mathrm{KBr}) v_{\max }\left(\mathrm{cm}^{-1}\right)$ : 3051, 2968, 1774, 1716; ${ }^{1} \mathrm{H}$ NMR $\left(\mathrm{CDCl}_{3}\right): \delta 1.11\left(3 \mathrm{H}, \mathrm{t}, J=7.1 \mathrm{~Hz}, C_{3}\right), 2.57(2 \mathrm{H}, \mathrm{t}, J=7.2$ $\left.\mathrm{Hz}, \mathrm{O}=\mathrm{CCH}_{2}\right), 3.86\left(2 \mathrm{H}, \mathrm{t}, J=7.2 \mathrm{~Hz}, \mathrm{NCH}_{2}\right), 4.06\left(2 \mathrm{H}, \mathrm{q}, J=7.1 \mathrm{~Hz}, \mathrm{OCH}_{2}\right), 7.57-7.70(4 \mathrm{H}$, $\mathrm{m}) ;{ }^{13} \mathrm{C} \mathrm{NMR}\left(\mathrm{CDCl}_{3}\right): \delta 13.8,32.7,33.5,64.3,122.9,131.7,133.8,167.5,170.6 ; \mathrm{MS} \mathrm{m} / \mathrm{z}(\%)$ : $247\left(\mathrm{M}^{+}, 20\right)$.

Butyl 3-phthalimido propanoate (1b). Colorless solid; mp 51-52 ${ }^{\circ} \mathrm{C}$; IR $(\mathrm{KBr}) v_{\max }\left(\mathrm{cm}^{-1}\right)$ : 3069, 2960, 1774, 1716; ${ }^{1} \mathrm{H}$ NMR $\left(\mathrm{CDCl}_{3}\right): \delta 0.90\left(3 \mathrm{H}, \mathrm{t}, J=6.8 \mathrm{~Hz}, \mathrm{CH}_{3}\right), 1.28(2 \mathrm{H}, \mathrm{m}$, $\left.\mathrm{CH}_{3} \mathrm{CH}_{2}\right), 1.59\left(2 \mathrm{H}, \mathrm{m}, \mathrm{CH}_{3} \mathrm{CH}_{2} \mathrm{CH}_{2}\right), 2.58\left(2 \mathrm{H}, \mathrm{t}, J=7.2 \mathrm{~Hz}, \mathrm{O}=\mathrm{CCH}_{2}\right), 3.91(2 \mathrm{H}, \mathrm{t}, J=7.2 \mathrm{~Hz}$, $\left.\mathrm{NCH}_{2}\right), 4.08\left(2 \mathrm{H}, \mathrm{t}, J=6.9 \mathrm{~Hz}, \mathrm{OCH}_{2}\right), 7.64-7.76(4 \mathrm{H}, \mathrm{m}) ;{ }^{13} \mathrm{C} \mathrm{NMR}\left(\mathrm{CDCl}_{3}\right): \delta 13.3,18.5$, $30.2,32.5,33.4,64.1,122.8,131.7,133.6,167.3,170.3 ; \mathrm{MS} \mathrm{m} / \mathrm{z}(\%): 275\left(\mathrm{M}^{+}, 16\right)$.

Hexyl 3-phthalimido propanoate (1c). Colorless solid; mp 40-41 ${ }^{\circ} \mathrm{C}$; IR $(\mathrm{KBr}) v_{\max }\left(\mathrm{cm}^{-1}\right)$ : 3055, 2970, 1774, 1716; ${ }^{1} \mathrm{H}$ NMR $\left(\mathrm{CDCl}_{3}\right): \delta 0.89\left(3 \mathrm{H}, \mathrm{t}, J=6.7 \mathrm{~Hz}, C_{3}\right), 1.29-1.36(6 \mathrm{H}, \mathrm{m})$, $1.60\left(2 \mathrm{H}, \mathrm{m}, \mathrm{CH}_{3}\left(\mathrm{CH}_{2}\right)_{3} \mathrm{CH}_{2}\right), 2.59\left(2 \mathrm{H}, \mathrm{t}, J=7.0 \mathrm{~Hz}, \mathrm{O}=\mathrm{CCH}_{2}\right), 3.95\left(2 \mathrm{H}, \mathrm{t}, J=7.0 \mathrm{~Hz}, \mathrm{NCH}_{2}\right)$, $4.09\left(2 \mathrm{H}, \mathrm{t}, J=6.9 \mathrm{~Hz}, \mathrm{OCH}_{2}\right), 7.68-7.83(4 \mathrm{H}, \mathrm{m}) ;{ }^{13} \mathrm{C} \mathrm{NMR}\left(\mathrm{CDCl}_{3}\right): \delta$ 13.7, 22.1, 25.4, 28.1, 30.2, 32.4. 33.4, 64.3, 122.7, 132.1, 133.6, 167.2, 170.2; MS m/z (\%): $303\left(\mathrm{M}^{+}, 27\right)$.

Benzyl 3-phthalimido propanoate (1d). Colorless solid; mp 57-59 ${ }^{\circ} \mathrm{C}$; IR $(\mathrm{KBr}) v_{\max }\left(\mathrm{cm}^{-1}\right)$ : $3061,2958,1774,1716 ;{ }^{1} \mathrm{H}$ NMR $\left(\mathrm{CDCl}_{3}\right): \delta 2.59\left(2 \mathrm{H}, \mathrm{t}, J=7.1 \mathrm{~Hz}, \mathrm{O}=\mathrm{CCH}_{2}\right), 3.86(2 \mathrm{H}, \mathrm{t}, J=$ $\left.\left.7.1 \mathrm{~Hz}, \mathrm{NCH}_{2}\right), 4.94(2 \mathrm{H}, \mathrm{s}, \mathrm{OCH})_{2}\right), 7.08-7.17(5 \mathrm{H}, \mathrm{m}), 7.52(2 \mathrm{H}, \mathrm{m}), 7.63(2 \mathrm{H}, \mathrm{m}) ;{ }^{13} \mathrm{C} \mathrm{NMR}$ $\left(\mathrm{CDCl}_{3}\right): \delta 32.9,33.7,66.6,122.6,128.2,128.5,129.4,131.9,133.9,135.6,167.8,170.6$; MS $\mathrm{m} / \mathrm{z}(\%): 309\left(\mathrm{M}^{+}, 19\right)$.

Phenethyl 3-phthalimido propanoate (1e). Colorless solid; mp 53-55 ${ }^{\circ} \mathrm{C}$; IR $(\mathrm{KBr}) v_{\max }\left(\mathrm{cm}^{-1}\right)$ : 3052, 2933, 1774, 1716; ${ }^{1} \mathrm{H}$ NMR $\left(\mathrm{CDCl}_{3}\right): \delta 2.69\left(2 \mathrm{H}, \mathrm{t}, J=7.2 \mathrm{~Hz}, \mathrm{O}=\mathrm{CCH}_{2}\right), 2.87(2 \mathrm{H}, \mathrm{t}, J=$ $\left.7.1 \mathrm{~Hz}, \mathrm{ArCH}_{2}\right), 3.93\left(2 \mathrm{H}, \mathrm{t}, J=7.2 \mathrm{~Hz}, \mathrm{NCH}_{2}\right), 4.26\left(2 \mathrm{H}, \mathrm{t}, J=7.1 \mathrm{~Hz}, \mathrm{OCH}_{2}\right), 7.13-7.26(5 \mathrm{H}$, m), $7.64(2 \mathrm{H}, \mathrm{m}), 7.77(2 \mathrm{H}, \mathrm{m}) ;{ }^{13} \mathrm{C} \mathrm{NMR}\left(\mathrm{CDCl}_{3}\right): \delta 32.8,33.6,34.9,65.2,123.1,126.4,128.3$, 128.9, 132.7, 133.9, 137.6, 167.8, 170.7; MS m/z (\%): $323\left(\mathrm{M}^{+}, 18\right)$.

Cinnamyl 3-phthalimido propanoate (1f). Pale yellow oil; IR (neat) $v_{\max }\left(\mathrm{cm}^{-1}\right): 3061,2954$, $1774,1716,1497 ;{ }^{1} \mathrm{H}$ NMR $\left(\mathrm{CDCl}_{3}\right): \delta 2.68\left(2 \mathrm{H}, \mathrm{t}, J=7.1 \mathrm{~Hz}, \mathrm{O}=\mathrm{CCH}_{2}\right), 3.92(2 \mathrm{H}, \mathrm{t}, J=7.1$ 
$\left.\mathrm{Hz}, \mathrm{NCH}_{2}\right), 4.64\left(2 \mathrm{H}, \mathrm{m}, \mathrm{OCH}_{2}\right), 6.12(1 \mathrm{H}, \mathrm{m}, \mathrm{ArCH}=\mathrm{CH}), 6.49(1 \mathrm{H}, \mathrm{d}, J=15.8 \mathrm{~Hz}, \mathrm{ArCH})$, 7.17-7.26 (5H, m), $7.57(2 \mathrm{H}, \mathrm{m}), 7.72(2 \mathrm{H}, \mathrm{m}) ;{ }^{13} \mathrm{C} \mathrm{NMR}\left(\mathrm{CDCl}_{3}\right): \delta 32.9,33.7,65.4,122.8$, 123.2, 126.6, 128.0, 128.5, 131.9, 133.9, 134.4, 136.1, 167.9, 170.6; MS m/z (\%): $335\left(\mathrm{M}^{+}, 14\right)$.

2-Hydroxy-3-(2-methoxyphenoxy)propyl 3-phthalimido propanoate (1g). Pale yellow oil; IR (neat) $v_{\max }\left(\mathrm{cm}^{-1}\right): 3480,3049,2948,1770,1715 ;{ }^{1} \mathrm{H} \mathrm{NMR}\left(\mathrm{CDCl}_{3}\right): \delta 2.63(2 \mathrm{H}, \mathrm{t}, J=7.0 \mathrm{~Hz}$, $\left.\mathrm{O}=\mathrm{CCH}_{2}\right), 3.67\left(3 \mathrm{H}, \mathrm{s}, \mathrm{CH}_{3}\right), 3.80-390(5 \mathrm{H}, \mathrm{m}), 4.11-4.19(3 \mathrm{H}, \mathrm{m}), 6.73-6.80(4 \mathrm{H}, \mathrm{m}), 7.54(2 \mathrm{H}$, $\mathrm{m}), 7.68(2 \mathrm{H}, \mathrm{m}) ;{ }^{13} \mathrm{C} \mathrm{NMR}\left(\mathrm{CDCl}_{3}\right): \delta 32.8,33.6,55.7,65.7,67.9,70.5,111.9,114.2,120.9$, $121.8,123.2$, 131.7, 134.0, 147.8, 149.3, 168.0, 170.8; MS m/z (\%): $399\left(\mathrm{M}^{+}, 20\right)$.

Phenyl 3-phthalimido propanoate (1h). Colorless solid; mp 70-72 ${ }^{\circ} \mathrm{C}$; IR ( $\left.\mathrm{KBr}\right) v_{\max }\left(\mathrm{cm}^{-1}\right)$ : 3076, 2970, 1775, 1713; ${ }^{1} \mathrm{H}$ NMR $\left(\mathrm{CDCl}_{3}\right): \delta 2.74\left(2 \mathrm{H}, \mathrm{t}, J=7.1 \mathrm{~Hz}, \mathrm{O}=\mathrm{CCH}_{2}\right), 3.95(2 \mathrm{H}, \mathrm{t}, J=$ $\left.7.1 \mathrm{~Hz}, \mathrm{NCH}_{2}\right), 6.98(2 \mathrm{H}, \mathrm{d}, J=8.0 \mathrm{~Hz}), 7.09(1 \mathrm{H}, \mathrm{d}, J=6.9), 7.24(2 \mathrm{H}, \mathrm{dd}, J=6.9,8.0 \mathrm{~Hz})$, 7.61-7.73 (4H, m); ${ }^{13} \mathrm{C} \mathrm{NMR}\left(\mathrm{CDCl}_{3}\right): \delta 32.9,33.5,121.4,122.9,125.7,129.2,131.6,133.9$, 150.3, 167.5, 170.3; MS m/z (\%): $295\left(\mathrm{M}^{+}, 2\right)$.

Naphthalen-2-yl 3-phthalimido propanoate (1i). Pale yellow solid; mp 88-90 ${ }^{\circ} \mathrm{C}$; IR $(\mathrm{KBr})$ $v_{\max }\left(\mathrm{cm}^{-1}\right): 3065,2958,1775,1716 ;{ }^{1} \mathrm{H}$ NMR $\left(\mathrm{CDCl}_{3}\right): \delta 2.69\left(2 \mathrm{H}, \mathrm{t}, J=7.2 \mathrm{~Hz}, \mathrm{O}=\mathrm{CCH}_{2}\right)$, $3.92\left(2 \mathrm{H}, \mathrm{t}, J=7.2 \mathrm{~Hz}, \mathrm{NCH}_{2}\right), 7.01-7.08(3 \mathrm{H}, \mathrm{m}), 7.21-7.31(3 \mathrm{H}, \mathrm{m}), 7.55-7.64(3 \mathrm{H}, \mathrm{m}), 7.76$ $(2 \mathrm{H}, \mathrm{m}) ;{ }^{13} \mathrm{C} \mathrm{NMR}\left(\mathrm{CDCl}_{3}\right): \delta 32.0,33.8,108.4,116.8,121.6,122.2,122.6,125.4,126.7,127.8$, $128.7,129.9,133.0,133.6,152.6,167.4,170.6 ; \mathrm{MS} \mathrm{m} / \mathrm{z}(\%): 345\left(\mathrm{M}^{+}, 3\right)$.

Butyl 3-(1,1-dioxido-3-oxo-1,2-benzisothiazol-2(3H)-yl)propanoate (2a). Yellow oil; IR (neat) $v_{\max }\left(\mathrm{cm}^{-1}\right): 3039,2938,1733,1455,1333 ;{ }^{1} \mathrm{H} \mathrm{NMR}\left(\mathrm{CDCl}_{3}\right): \delta 0.84(3 \mathrm{H}, \mathrm{t}, J=7.4 \mathrm{~Hz}$, $\left.\mathrm{CH}_{3}\right), 1.25\left(2 \mathrm{H}, \mathrm{m}, \mathrm{CH}_{3} \mathrm{CH}_{2}\right), 1.51\left(2 \mathrm{H}, \mathrm{m}, \mathrm{CH}_{3} \mathrm{CH}_{2} \mathrm{CH}_{2}\right), 2.80\left(2 \mathrm{H}, \mathrm{t}, J=6.8 \mathrm{~Hz}, \mathrm{O}=\mathrm{CCH}_{2}\right)$, 4.00-4.08 (4H, m, $\mathrm{NCH}_{2}$ and $\left.\mathrm{OCH}_{2}\right), 7.81-7.97(4 \mathrm{H}, \mathrm{m}) ;{ }^{13} \mathrm{C} \mathrm{NMR}\left(\mathrm{CDCl}_{3}\right): \delta 12.6,18.0,29.4$, $32.0,33.6,63.9,119.9,124.2,126.2,133.3,133.8,136.4,157.6,169.3 ; \mathrm{MS} \mathrm{m} / \mathrm{z}(\%): 311\left(\mathrm{M}^{+}, 1\right)$, 247( $\left.\mathrm{M}^{+}-\mathrm{SO}_{2}, 21\right)$.

Hexyl 3-(1,1-dioxido-3-oxo-1,2-benzisothiazol-2(3H)-yl)propanoate (2b). Pale yellow oil; IR (neat) $v_{\max }\left(\mathrm{cm}^{-1}\right): 3051,2931,1734,1457,1339 ;{ }^{1} \mathrm{H} \mathrm{NMR}\left(\mathrm{CDCl}_{3}\right): \delta 0.78(3 \mathrm{H}, \mathrm{t}, J=7.0 \mathrm{~Hz}$, $\left.\mathrm{CH}_{3}\right), 1.16-1.25(6 \mathrm{H}, \mathrm{m}), 1.53\left(2 \mathrm{H}, \mathrm{m}, \mathrm{CH}_{3}\left(\mathrm{CH}_{2}\right)_{3} \mathrm{CH}_{2}\right), 2.81\left(2 \mathrm{H}, \mathrm{t}, \mathrm{J}=6.7 \mathrm{~Hz}, \mathrm{O}=\mathrm{CCH}_{2}\right), 3.95-$ $4.04\left(4 \mathrm{H}, \mathrm{m}, \mathrm{NCH}_{2}\right.$ and $\left.\mathrm{OCH}_{2}\right), 7.78-7.92(4 \mathrm{H}, \mathrm{m}) ;{ }^{13} \mathrm{C} \mathrm{NMR}\left(\mathrm{CDCl}_{3}\right): \delta 12.9,21.4,24.4,27.4$, $30.3,32.0,33.6,64.1,119.9,124.1,126.1,133.4,133.9,136.6,157.6,169.2 ; \mathrm{MS} \mathrm{m} / \mathrm{z}(\%): 339$ $\left(\mathrm{M}^{+}, 1\right), 275\left(\mathrm{M}^{+}-\mathrm{SO}_{2}, 30\right)$.

Cinnamyl 3-(1,1-dioxido-3-oxo-1,2-benzisothiazol-2(3H)-yl)propanoate (2c). Yellow oil; IR (neat) $v_{\max }\left(\mathrm{cm}^{-1}\right): 3061,2954,1733,1338,1303,1182 ;{ }^{1} \mathrm{H} \mathrm{NMR}\left(\mathrm{CDCl}_{3}\right): \delta 2.85(2 \mathrm{H}, \mathrm{t}, J=6.9$ $\left.\mathrm{Hz}, \mathrm{O}=\mathrm{CCH}_{2}\right), 4.03\left(2 \mathrm{H}, \mathrm{t}, J=6.9 \mathrm{~Hz}, \mathrm{NCH}_{2}\right), 4.70\left(2 \mathrm{H}, \mathrm{m}, \mathrm{OCH}_{2}\right), 6.23(1 \mathrm{H}, \mathrm{m}, \mathrm{ArCH}=\mathrm{CH})$, $6.54(1 \mathrm{H}, \mathrm{d}, J=15.7 \mathrm{~Hz}, \operatorname{ArCH}), 7.19-7.30(5 \mathrm{H}, \mathrm{m}), 7.76-7.93(4 \mathrm{H}, \mathrm{m}) ;{ }^{13} \mathrm{C} \mathrm{NMR}\left(\mathrm{CDCl}_{3}\right): \delta$ $32.06,33.6,64.5,119.9,121.7,124.2,125.6,126.1,126.7,127.0,127.5,133.3,133.5,133.8$, 136.8, 157.5, 168.5; MS m/z (\%): $307\left(\mathrm{M}^{+}-\mathrm{SO}_{2}, 16\right)$.

Isopropyl 3-(1,1-dioxido-3-oxo-1,2-benzisothiazol-2(3H)-yl)propanoate (2d). Pale yellow oil; IR (neat) $v_{\max }\left(\mathrm{cm}^{-1}\right): 3033,2982,1734,1457,1340 ;{ }^{1} \mathrm{H} \mathrm{NMR}\left(\mathrm{CDCl}_{3}\right): \delta 1.18(6 \mathrm{H}, \mathrm{d}, J=6.6$ $\left.\mathrm{Hz}, 2 \mathrm{CH}_{3}\right), 2.77\left(2 \mathrm{H}, \mathrm{t}, J=6.7, \mathrm{O}=\mathrm{CCH}_{2}\right), 4.01\left(2 \mathrm{H}, \mathrm{t}, J=6.7 \mathrm{~Hz}, \mathrm{NCH}_{2}\right), 4.97(1 \mathrm{H}, \mathrm{q}, J=6.6$, 
$\mathrm{OCH}), 7.79-7.95(4 \mathrm{H}, \mathrm{m}) ;{ }^{13} \mathrm{C} \mathrm{NMR}\left(\mathrm{CDCl}_{3}\right): \delta 20.7,32.2,33.6,67.5,119.9,124.2,126.2$, 133.3, 133.8, 136.6, 157.6, 168.7; MS m/z (\%): $297\left(\mathrm{M}^{+}, 2\right), 233\left(\mathrm{M}^{+}-\mathrm{SO}_{2}, 38\right)$.

Cyclohexyl 3-(1,1-dioxido-3-oxo-1,2-benzisothiazol-2(3H)-yl)propanoate (2e). Colorless solid; mp 62-64 ${ }^{\circ} \mathrm{C}$; IR (neat) $v_{\max }\left(\mathrm{cm}^{-1}\right): 2938,1733,1339 ;{ }^{1} \mathrm{H}$ NMR $\left(\mathrm{CDCl}_{3}\right): \delta 1.19-1.36(6 \mathrm{H}$, m), 1.65-1.81 (4H, m), $2.81\left(2 \mathrm{H}, \mathrm{t}, J=6.8 \mathrm{~Hz}, \mathrm{O}=\mathrm{CCH}_{2}\right), 4.04\left(2 \mathrm{H}, \mathrm{t}, J=6.8 \mathrm{~Hz}, \mathrm{NCH}_{2}\right), 4.76$ $(1 \mathrm{H}, \mathrm{m}, \mathrm{OCH}), 7.81-8.00(4 \mathrm{H}, \mathrm{m}) ;{ }^{13} \mathrm{C} \mathrm{NMR}\left(\mathrm{CDCl}_{3}\right): \delta 23.6,25.2,31.4,33.2,34.6,73.3,120.9$, 125.1, 127.1, 134.3, 134.8, 137.5, 158.6, 169.6; $\mathrm{MS} \mathrm{m} / \mathrm{z}(\%): 273\left(\mathrm{M}^{+}-\mathrm{SO}_{2}, 33\right)$.

\section{Acknowledgements}

We appreciate Payam Noor University of Bushehr, Persian Gulf University and Shiraz University research councils for financial support of this work. We are also grateful to Prof. H. Sharghi for helpful discussion and to Mr. H. Sajedian Fard for running the NMR spectra.

\section{References and Footnotes}

1. Norman, M. H.; Minick, D. J.; Rigdon, G. C. J. Med. Chem. 1996, 39, 149.

2. (a) Lima, L. M.; Castro, P.; Machado, A. L.; Fraga, C. A. M.; Lugnier, C.; de Moraesc, V. L. G.; Barreiroa, E. J. Bioorg. Med. Chem. 2002, 10, 3067. (b) Collin, X.; Robert, J. M.; Wielgosz, G.; Le Baut G.; Grimaud, N.; Petit, J. Y. Eur. J. Med. Chem. 2001, 36, 639.

3. Raasch, A.; Scharfestein, O.; Trankle, C.; Holzgrabe, U.; Mohr, K. J. Med. Chem. 2002, 45, 3809.

4. Chapman, J. M.; Cocolas, G. H.; Hall, I. H. J. Med. Chem. 1983, 26, 243.

5. (a) Soler, L.; Cerrada, V.; Matía, M. P.; Novella, J. L.; Alvarez-Builla, J. Arkivoc 2007 (iv) 312. (b) Güzel, Ö.; Salman, A. Bioorg. Med. Chem. 2006, 14, 7804. (c) Filho, J. C.; Santini, A. O.; Nasser A. L. M. Food chemistry 2003, 83, 297. (d) Price, M. J.; Biava, G. C.; Oser, L. B.; Vogin, E. E.; Steinfeld, J.; Ley, L. H. Science 1970, 167, 1131. (e) Weihrauch, M. R.; Diehl, V.; Bohlen, H. Mediz. Klin. 2001, 96, 670. (f) Squire, R. A. Food Chem. Toxicol. 1985, 23, 491. (g) Cohen, S. M.; Garland, E. M.; Cano, M.; John, M. K.; Khanchab, M.; Wehner, J. M.; Arnold, L. L. Carcinogenesis 1995, 16, 2743. (h) Takayama, S.; Sieber, S. M.; Adamson, R. H.; Thorgeirsson, U. P.; Dalgard, D. W.; Arnold, L. L.; Cano, M.; Eklund, S.; Cohen, S. M. J. Natl. Cancer Inst. 1998, 90,19 (i) Jensen, O. M.; Kamby, C. Int. J. Cancer 1982, 29, 507. (j) Cohen, S. M. Toxicology 1995, 102, 149. (k) Zurlo, J.; Squire, R. A. J. Natl. Cancer Inst. 1998, 90, 2. (1) Turner, S. D.; Tinwell, H.; Piegosrch, W.; Schmerzer, P.; Ashby, J.; Muta-genesis 2001, 16, 329. (m) Malik, K. M. A.; Haider, S. Z.; Hossain, M. A.; Hursthouse, M. B. Acta Crystallogr. C 1984, 40, 1696.

6. Baran, E. J.; Yilmaz, V. T. Coordination Chem. Rev. 2006, In press.

7. Gellman, S. H. Acc. Chem. Res. 1998, 31, 173. 
8. Shinagawa, S.; Kanamaru, T.; Harada, S.; Asai, M.; Okazaki H. J. Med. Chem. 1987, 30, 1458.

9. Windholz, M.; Budavari, S.; Stroumtsos, L. Y.; Fertig, M. N. The Merck Index, An Encyclopedia of Chemicals and Drugs, $9^{\text {th }}$ ed.; Merck, Rahway, NJ, 1976, p. 909.

10. Shih, C.; Gossett, L. S.; Gruber, J. M.; Grossman, C. S.; Andis, S. L.; Schultz, R. M.; Worzalla, J. F.; Corbett, T. H.; Metz, J. T. Bioorg. Med. Chem. Lett. 1999, 9, 69.

11. Yuan, C.; Williams, R. M. J. Am. Chem. Soc. 1997, 119, 11777.

12. (a) Ahmed, N.; van Lier, J. E. Tetrahedron Lett. 2007, 48, 13. (b) Ranu, B. C.; Banerjee, S. Tetrahedron Lett. 2007, 48, 141. (c) Łyżwa, P.; Jankowiak, A.; Kwiatkowska, M.; Mikołajczyk, M.; Kiełbasiński, P.; Betz, A.; Jaffres, P.-A.; Gaumont, A.-C.; Gulea, M. Tetrahedron Lett. 2007, 48, 351. (d) Claramunt, R. M.; Sanz, D.; Aggarwal, S.; Kumar, A.; Prakash, O.; Singh, S. P.; Elgueroc, J. Arkivoc 2006 (xiv) 35. (e) Fetterly, B. M.; Jana, N. K.; Verkade, J. G. Tetrahedron 2006, 62, 440. (f)Yu, A.; Yenil, R. N.; Pesquet, A.; Oulyadi, H.; Maddaluno, J. Tetrahedron 2006, 62, 5411. (g) Surendra, K.; Krishnaveni, N. S.; Sridhar, R.; Rao, K. R. Tetrahedron Lett. 2006, 47, 2125. (h) Naidu, B. N.; Li, W.; Sorenson, M. E.; Connolly, T. P.; Wichtowski, J. A.; Zhang, Y.; Kim, O. K.; Matiskella, J. D.; Lam, K. S.; Bronson, J. J.; Ueda, Y. Tetrahedron Lett. 2004, 45, 1059. (i) Makara, G. M.; Ma, Y. Tetrahedron Lett. 2001, 42, 4123. (j) Ranu, B. C.; Dey, S. S.; Hajra, A. Arkivoc 2002 (vii) 76. (k) Prabagaran, N.; Abraham, S.; Sundararajan, G. Arkivoc 2002 (vii) 212. (1) Wabnitz, T. C.; Spencer, J. B. Org. Lett. 2003, 2141. (m) Shaikh, N. S.; Deshpande, V. H.; Bedekar, A. V. Tetrahedron 2001, 57, 9045. (n) Cabral, J.; Laszlo, P.; Mahè, L.; Montaufier, M.-T.; Randriamahefà, S. L. Tetrahedron Lett. 1989, 30, 3969.

13. Azizi, N.; Saidi, M. R. Tetrahedron 2004, 60, 383.

14. (a) Moe, O. A.; Warner, D. T. J. Am. Chem. Soc. 1949, 71, 1251. (b) Raiman, M. V.; Pukin, A. V.; Tyvorskii, V. I.; Kimpe, N. D.; Kulinkovich, O. G. Tetrahedron 2003, 59, 5265. (c) Cardillo, G.; Simone, A. D.; Gentilucci, L.; Sabatino, P.; Tomasini, C. Tetrahedron Lett. 1994, 35, 5051.

15. Varma, R. S. Tetrahedron 2002, 58, 1235.

16. (a) Sharghi, H.; Hosseini, M. Synthesis 2002, 1057. (b) Hosseini Sarvari, M.; Sharghi, H. J. Org. Chem. 2004, 69, 6953. (c) Gupta, M.; Paul, S.; Gupta, R.; Loupy, A., Tetrahedron Lett. 2005, 46, 4957. (d) Hosseini Sarvari, M. Synthesis 2005, 787. (e) Kim, Y. J.; Varma, R. S., Tetrahedron Lett. 2004, 45, 7205. (f) Hosseini Sarvari, M.; Sharghi, H. Tetrahedron 2005, 61, 10903. (g) Tamaddon, F.; Amrollahi, M. A.; Sharafat, L. Tetrahedron Lett. 2005, 46, 7841.

17. (a) Dewan, S. K.; Singh. R.; Kumar, A. Arkivoc 2006 (ii) 41. (b) Zou, X.; Tu, S.; Shi, F.; Xu, J. Arkivoc 2006 (ii) 130. (c) Habibi, D.; Marvi, O. Arkivoc 2006 (xiii) 8. (d) Reddy, P. B.; Singh, P. P.; Sawant, S. D.; Koul, S.; Taneja, S. C.; Kumar, H. M. S. Arkivoc 2006 (xiii) 142. (e) Nadaraj, V.; Selvi, S. T.; Sasi, R. Arkivoc 2006 (v) 82. (f) Rajithaa, B.; Kumara, V. N.; Someshwara, P.; Madhava, J. V.; Reddy, P. N.; Reddyb, Y. T. Arkivoc 2006 (xii) 23. (g) Mojtahedi, M. M.; Ghasemi, M. H.; Abaee, M. S.; Bolourtchian, M. Arkivoc 2005 (xv) 68. 
(h) Rábarová, E.; Koiš, P.; Lácová, M.; Krutošíková, A. Arkivoc 2004 (i) 110. (i) Kappe, C. O. Angew. Chem., Int. Ed. 2004, 43, 6250. (j) Loupy, A. Microwaves in Organic Synthesis, Wiley-VCH, Weinheim, 2004. (k) Wang, G.-W.; Cheng, B. Arkivoc 2004 (ix) 4. (1) Katritzky, A. R.; Singh, S. K. Arkivoc 2003 (xiii) 68. (m) Lidstrom, P.; Tierney, J.; Wathey, B.; Westman, J. Tetrahedron 2001, 57, 9225. (n) Kabalka, G. W.; Wang, L.; Pagni, R. M. Arkivoc 2001 (iv) 5. (o) De la Hoz, A.; Diaz-Ortis, A.; Moreno, A.; Langa, F. Eur. J. Org. Chem. 2000, 3659. (p) Caddick, S. Tetrahedron 1995, 57, 10403. (q) Varma, R. S. Green Chem. 1999, 1, 43. (r) Loupy, A.; Petit, A.; Hamelin, J.; Texier-Boullet, F.; Jacquault, P.; Mathe, D. Synthesis 1998, 1213. (s) Katritzky, A. R.; Vincek, A. S.; Suzuki, K. Arkivoc 2005 (v) 116.

18. (a) Khalafi-Nezhad, A.; Zarea, A.; Soltani Rad, M. N.; Mokhtari, B.; Parhami, A. Synthesis 2005, 419. (b) Khalafi-Nezhad, A.; Zare, A.; Parhami, A.; Soltani Rad, M. N.; Nejabat, G. R. Synth. Commun. 2006, 36, In press.

19. (a) Khalafi-Nezhad, A.; Zare, A.; Parhami, A.; Soltani Rad, M. N. Arkivoc 2006 (xii) 161. (b) Khalafi-Nezhad, A.; Parhami, A.; Soltani Rad, M. N.; Zare, A. Tetrahedron Lett. 2005, 46, 6879. (c) Khalafi-Nezhad, A.; Soltani Rad, M. N.; Khoshnood, A. Synthesis 2004, 583. (d) Khalafi-Nezhad, A.; Mokhtari, B. Tetrahedron Lett. 2004, 45, 6737. (e) Khalafi-Nezhad, A.; Mokhtari, B.; Soltani Rad, M. N. Tetrahedron Lett. 2003, 44, 7325. (f) Khalafi-Nezhad, A.; Alamdari, F.; Zekri, N. Tetrahedron 2000, 56, 7503. (g) Khalafi-Nezhad, A.; Hashemi, A. J. Chem. Res. (S) 1999, 720.

20. Wasserscheid, P.; Welton, T. Ionic Liquids in Synthesis, Wiley-VCH: Weinheim, 2002. 